\title{
Effects of Ground-Plane Size on Planar UWB Monopole Antenna
}

\author{
Y. F. Weng, S. W. Cheung and T. I. Yuk \\ Department of Electrical and Electronic Engineering \\ The University of Hong Kong, Hong Kong \\ E-mail: [yfweng, swcheung, tiyuk]@eee.hku.hk
}

\begin{abstract}
Planar monopole antennas have found widespread applications in wireless communication systems. Their advantages of compact size, omnidirectional radiation pattern and wide impedance bandwidth make them good candidates for the designs of ultra-wideband (UWB) antennas. This paper investigates the effects of the dimensions of the rectangular ground-plane on the return loss and efficiency of an elliptical planar monopole antenna for UWB applications. Computer simulation and measurement are carried out on a group of nine antennas with different rectangular ground plane sizes. The simulated and measured results on return loss agree well. However, the simulated and measured efficiencies do not agree well in the lower UWB band. The measured efficiency shows that the efficiency is affected more by the width rather than by the length of the ground plane while the length of the ground plane affects the lower cutoff frequency.
\end{abstract}

Keywords - planar monopole antenna, ultra-wideband (UWB) antenna, ground plane effects, ground-plane size.

\section{INTRODUCTION}

Since the allocation of the 3.1-10.6 GHz frequency band for Ultra-wideband (UWB) communication systems by the USFCC in 2002 [1], researches on UWB technologies have attracted a lot of attentions in both the commercial and academic domains. In most UWB applications such as PCs, high-definition TVs and digital cameras etc., compact and low cost UWB antennas with non-dispersive and omnidirectional radiation pattern are desirable. Planar monopole antennas have the advantages of low-profile, ease of fabrication on printed circuits board and ease of integration with other RF components, and so are more favorable to the traditional 3-D antennas for compact UWB applications.

In general, a monopole antenna consists of a radiator (which could have many different types) fed by a microstrip line or coplanar waveguide (CPW) and a finite ground plane. A true ground should have no effect on the performance of the antenna, and so ideally the performance of the antenna should be independent of the ground-plane size. However, studies have shown that the ground plane with a finite size could also be a radiating element and the current distribution on the ground plane could affect the impedance bandwidth, gain and radiation pattern of the antenna [2-7]. Since the UWB antenna is ultimately mounted on a wireless device which may serve as part of the ground plane, the ground plane dependency brings difficulties to the design of UWB antennas for wireless devices. Different wireless devices will need different designs. Several methods have been proposed to reduce the ground plane dependence of the monopole antenna. In [5], loading two sleeves around the monopole antenna was used to widen the impendence bandwidth of the antenna and also achieve stable impedance matching for a large range of ground-plane size. In [6], the leakage blocking slots were used to partially prevent the current on the ground plane from flowing back to the outer conductor of the cable, and hence reducing the ground plane effects of the antenna. In [7], a swan-shaped antenna was proposed to greatly reduce the overall size of the antenna while keeping the antenna efficiency at a high level. However, little studies have been carried out on the effects of the size and dimension of the ground plane on antennas.

In this paper, we study the effects of size and dimension of a rectangular ground plane on the performance, in terms of return loss and efficiency, of a planar UWB monopole antenna. A group of nine antennas of the same type are used for studies. All these antennas have identical elliptical-shape radiator of the same size, but different rectangular ground plane sizes. Their dimensions are optimized using the CST MICROWAVE STUDIO (MS) software [8] to provide wide impedance bandwidths, covering the entire UWB band with return loss larger than $10 \mathrm{~dB}$. These antennas are also fabricated on PCBs and the performances in terms of return loss and efficiency are measured using the Satimo Starlab measurement system.

\section{ANTENNA USED FOR STUDIES}

A planar monopole antenna is selected for use to investigate the effects of different ground-plane sizes on the return loss and efficiency of the antenna. Figure 1 shows the antenna which consists of an elliptical radiator fed by a $50-\Omega$ microstrip line and a small rectangular ground-plane with size of $g l \times g w=15$ $\mathrm{mm} \times 30 \mathrm{~mm}$ on the other side of the substrate. The substrate used is the Rogers PCB, RO4350B, with a relative dielectric constant $\varepsilon_{r}=3.48$, a thickness $0.762 \mathrm{~mm}$ and a loss tangent 0.0037 . The width of the microstrip line linearly tapers from $w_{I}$ $=1.73 \mathrm{~mm}$ to $w_{2}=0.6 \mathrm{~mm}$ to achieve good impedance matching. The distance, gap, between the elliptical radiator and the upper edge of the ground is also critical for impedance matching and so has been optimized using computer simulation to be $0.3 \mathrm{~mm}$. Detailed dimensions of the antenna are listed in Table 1 . 


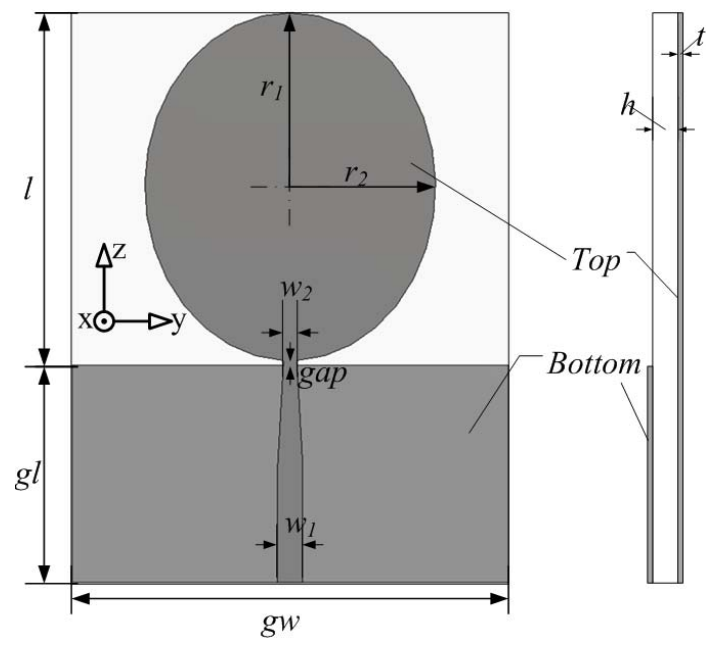

(a)

Fig. 1 Layout of the UWB antenna, (a) top view and (b) side view.

TABLE I. ANTENNA DIMENSIONS

\begin{tabular}{|c|c|c|c|}
\hline Parameter & Value(mm) & Parameter & Value(mm) \\
\hline$g l$ & 15 & $g w$ & 30 \\
\hline$h$ & 0.762 & $t$ & 0.035 \\
\hline$l$ & 24.3 & $g a p$ & 0.3 \\
\hline$r_{l}$ & 12 & $r_{2}$ & 9 \\
\hline
\end{tabular}

\section{RESUlTS AND DisCUSSIONS}

To study the effects of the ground-plane dimensions on the performance of a planar monopole antenna, a group of nine planar monopole antennas with identical elliptical radiator, as shown in Fig. 1, but different ground-plane sizes, is use for studies. The performance of each of these antennas is optimized using its dimensions through the use of the CST MW software. These antennas have also been fabricated on the Rogers PCBs, RO4305B, and one of the prototypes is shown in Fig. 2. The return losses and efficiencies of these antennas across the UWB band have also been measured by using the Satimo Starlab measurement system. In these measurements, a cable with choke was used to connect the antenna to the Satimo Starlab measurement system to reduce the current flow in the outer conductor of the cable and hence to reduce the cable effects. The simulated and measured return losses and efficiencies of these antennas with nine different ground-plane sizes across the UWB band are shown in Figs. 3(a) to 3(i) for comparison.

In these figures, a dash-dotted line and a dotted line are used to indicate the $50 \%$ efficiency and $10 \mathrm{~dB}$ return loss, respectively. It can be seen that there are good agreements between the simulated and measured return loss in all these nine antennas. Better agreements are obtained in the higher frequency band, especially above $6 \mathrm{GHz}$.

The simulated efficiencies in Fig. 3 show that, in all these cases, the measured results are always lower than the simulated results. This is expected because simulation tests assume the ideal case and do not take into account the connector, which is

not the case in practice. The antennas with larger ground-plane sizes have higher antenna efficiency. The discrepancies between the simulated and measured efficiencies are larger with smaller ground-plane sizes and at lower frequencies. Moreover, the width of the ground plane affects the efficiency more than the length. It is also noted that increasing the length of the ground plane lowers the lower cut off frequency of the antenna.

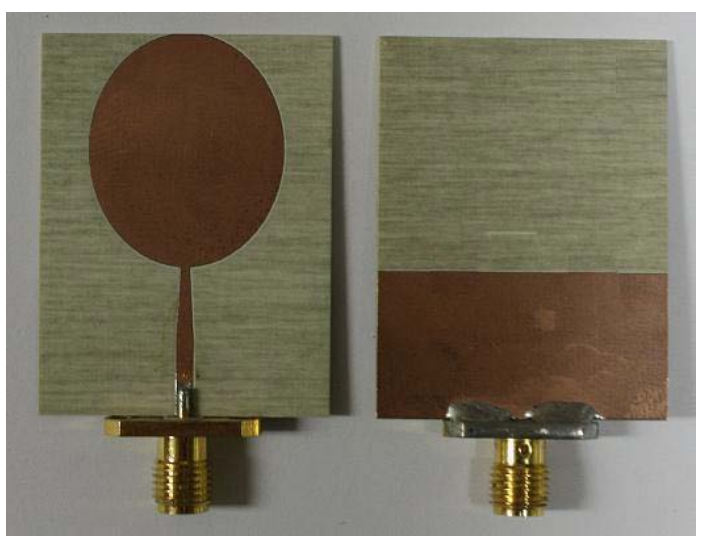

Fig. 2 Photograph of planar UWB monopole antenna.

Although the simulated and measured return loss agree well, it is not the case for the antenna efficiencies. The results in Fig. 3 show that, with smaller ground planes, the measured results of efficiencies are much lower than the simulated results at lower frequencies. It appears that the simulated results are less accurate in the low frequency band.

The discrepancy between the measured and simulated efficiencies gets smaller at higher frequencies. To better understand the effects of the ground plane dimensions on this discrepancy, we compare the averages of the discrepancy across the whole UWB band from 2 to $12 \mathrm{GHz}$ for all these nine antennas. Here, we divide the whole frequency band into three smaller sub-bands, 2-4 GHz, 4-6 GHz and 6-12 GHz. In each of these sub-bands, we calculate the average of the discrepancy as the arithmetic means of the absolute differences of the simulated and measured return losses. Results are listed in Table II. It is noted that the smaller the average discrepancy, the more accurate is the simulated results. It also can be seen in Table II that, the average discrepancies are smaller with larger ground-plane sizes and at higher frequencies. To have less discrepancy and hence more accurate simulated results across the whole frequency band, the ground plane size needs to be quite large. However, in the high frequency sub-band, the discrepancy is also small with small ground-plane sizes.

From Fig. 3 (a), (b) and (c), it can be seen that if the width of the ground plane increases from $30 \mathrm{~mm}$ to $80 \mathrm{~mm}$, while the length of the ground plane remains at $15 \mathrm{~mm}$, the discrepancies between the simulated and measured efficiencies becomes smaller and smaller. A detail numerical comparison could be found in the first row of Table II. The overall measured average efficiency discrepancies from $2-12 \mathrm{GHz}$ are $0.110,0.074$ and 0.040 with ground-plane size of $15(g l) \times 30(g w), 15(g l) \times 50$ $(g w)$ and $15(g l) \times 80(g w)$, respectively. The same descending trends could also be observed in the first row of Table II for the three sub-bands individually. However, if the width of the 
ground plane remains at $30 \mathrm{~mm}$ and the length of the groundplane increases from $15 \mathrm{~mm}$ to $50 \mathrm{~mm}$, as shown in Fig. 3 (a), (d) and (g), the discrepancies between the simulated and measured efficiencies are about at the same level for the overall average and all the three sub-bands averages. Furthermore, by comparing the return losses of Fig. 3 (a), (b) and (c), the lower cut-off frequencies of the three antennas are all around $2.8 \mathrm{GHz}$. While comparing the return loss of the Fig. 3 (a), (d) and (g), the lower cut-off frequencies of the three antennas are decreasing with values of $2.76,2.38$ and $2.21 \mathrm{GHz}$, respectively. Similar phenomena could also be easily observed for the figure groups of Fig. 3 (d), (e) and (f), Fig. 3 (b), (e) and (h), Fig. 3 (g), (h) and (i) and Fig. 3 (c), (f) and (i). Thus, we can conclude that the width of the ground plane has more effects on the efficiency than the length. Also, increasing the length of the ground plane lowers the lower cut off frequency of the antenna.

TABLE II. AVERAGE DisCREPANCY BETWEEN SimUlated AND MEASURED EFFICIENCIES

\begin{tabular}{|c|c|c|c|c|c|c|c|c|}
\hline \multicolumn{3}{|c|}{ Avg. 2-12 GHz } & \multicolumn{3}{|c|}{ Avg. 2-12 GHz } & \multicolumn{3}{|c|}{ Avg. 2-12 GHz } \\
\hline $2-4 \mathrm{GHz}$ & $4-6 \mathrm{GHz}$ & 6-12GHz & $2-4 \mathrm{GHz}$ & 4-6GHz & 6-12GHz & $2-4 \mathrm{GHz}$ & $4-6 \mathrm{GHz}$ & 6-12GHz \\
\hline \multicolumn{3}{|c|}{$15(g l) \times 30(g w)$} & \multicolumn{3}{|c|}{$15(g l) \times 50(g w)$} & \multicolumn{3}{|c|}{$15(g l) \times 80(g w)$} \\
\hline \multicolumn{3}{|c|}{0.110} & \multicolumn{3}{|c|}{0.074} & \multicolumn{3}{|c|}{0.040} \\
\hline 0.244 & 0.121 & 0.060 & 0.172 & 0.026 & 0.055 & 0.050 & 0.051 & 0.034 \\
\hline \multicolumn{3}{|c|}{$\begin{array}{c}30(g l) \times \mathbf{3 0}(\mathbf{g} w) \\
0.102\end{array}$} & \multicolumn{3}{|c|}{$\begin{array}{c}\mathbf{3 0}(\mathrm{gl}) \times \mathbf{5 0}(\mathbf{g w}) \\
0.081\end{array}$} & \multicolumn{3}{|c|}{$30(g l) \times 80(g w)$} \\
\hline 0.225 & 0.114 & 0.055 & 0.180 & 0.056 & 0.054 & 0.050 & 0.052 & 0.038 \\
\hline \multicolumn{3}{|c|}{$50(g l) \times 30(g w)$} & \multicolumn{3}{|c|}{$50(g l) \times 50(g w)$} & \multicolumn{3}{|c|}{$50(g l) \times 80(g w)$} \\
\hline \multicolumn{3}{|c|}{0.151} & \multicolumn{3}{|c|}{0.072} & \multicolumn{3}{|c|}{0.061} \\
\hline 0.186 & 0.116 & 0.058 & 0.130 & 0.057 & 0.056 & 0.069 & 0.058 & 0.061 \\
\hline
\end{tabular}

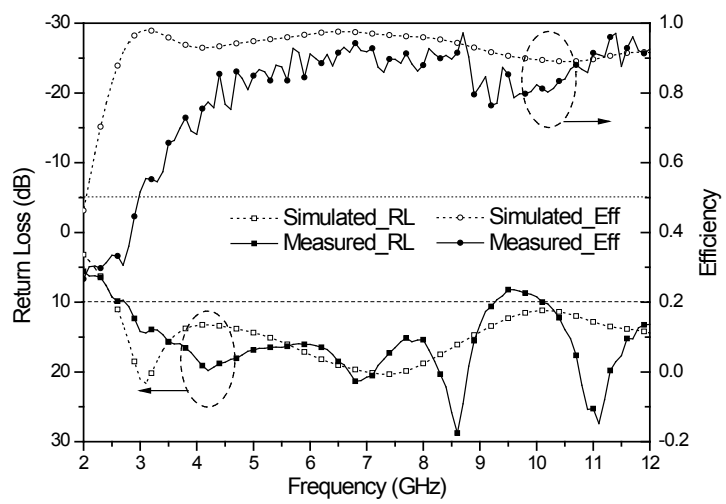

(a) $15(g l) * 30(g w)$

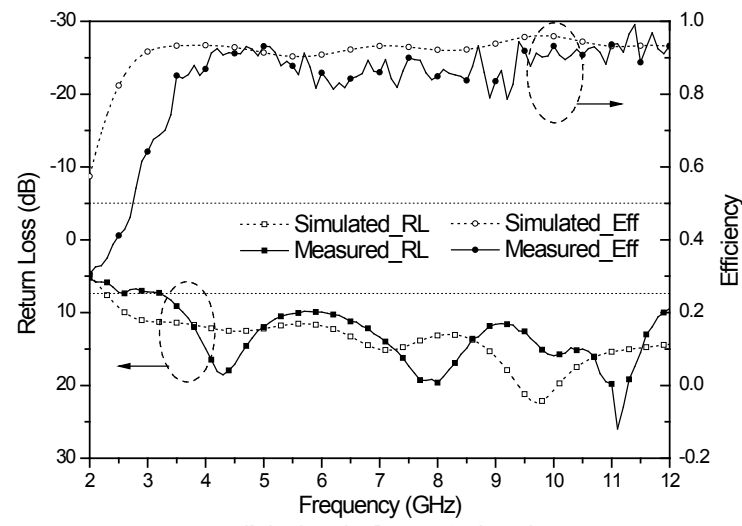

(b) $15(g l) * 50(g w)$

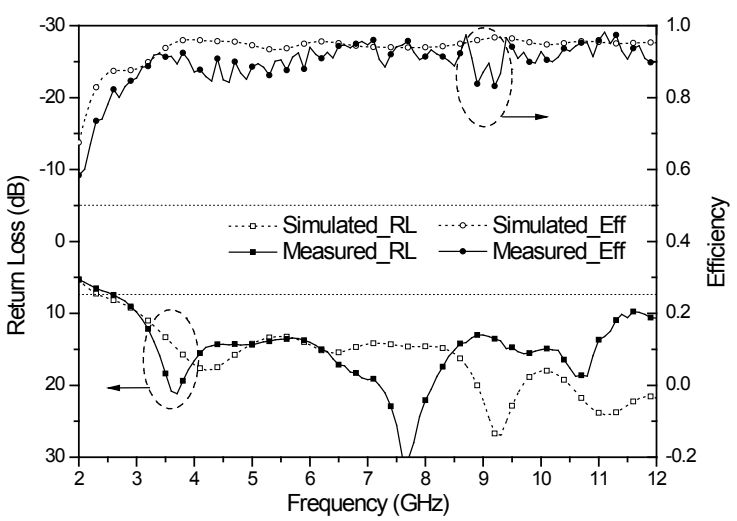

(c) $15(g l) * 80(g w)$

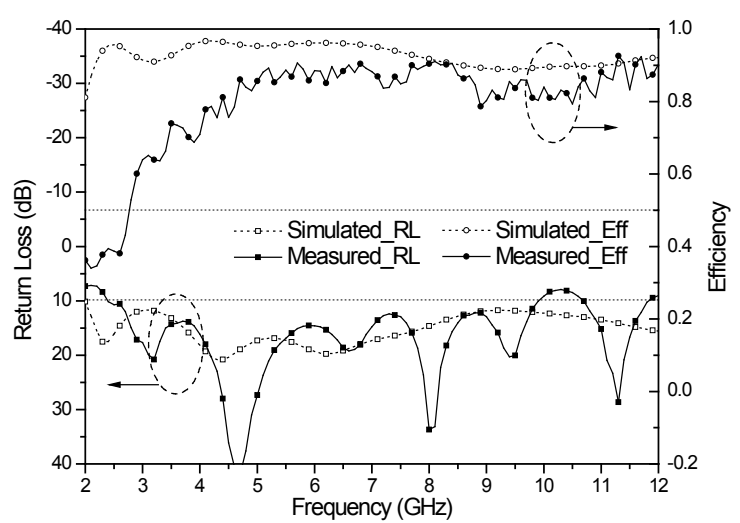

(d) $30(g l) * 30(g w)$

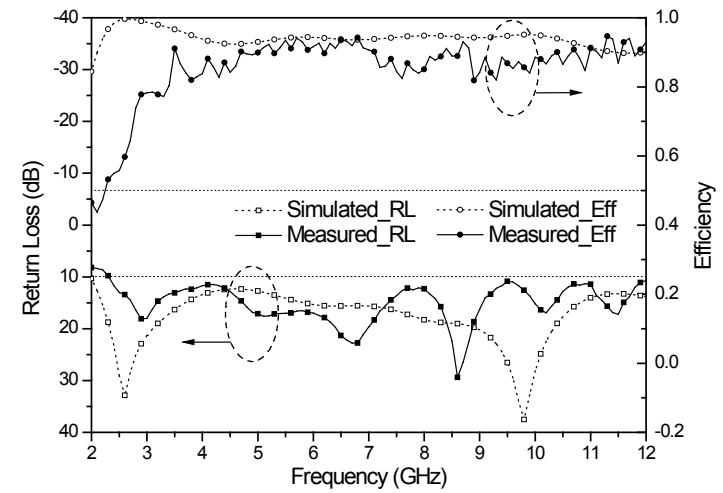

(e) $30(g /) * 50(g w)$

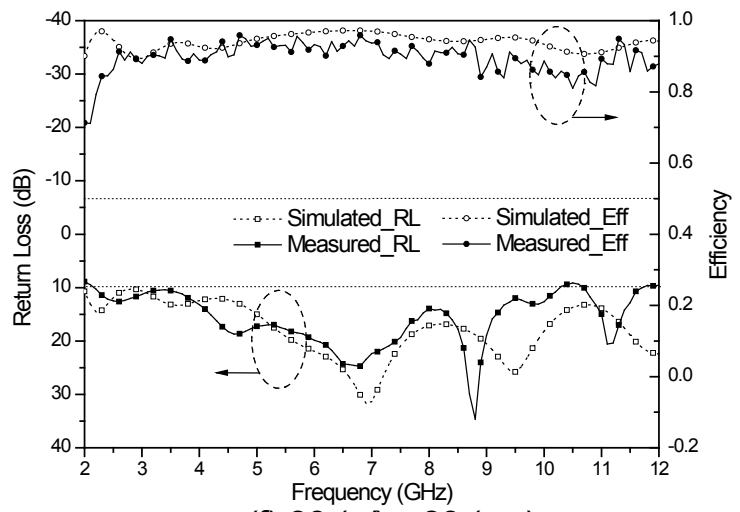

(f) $30(g l) * 80(g w)$ 


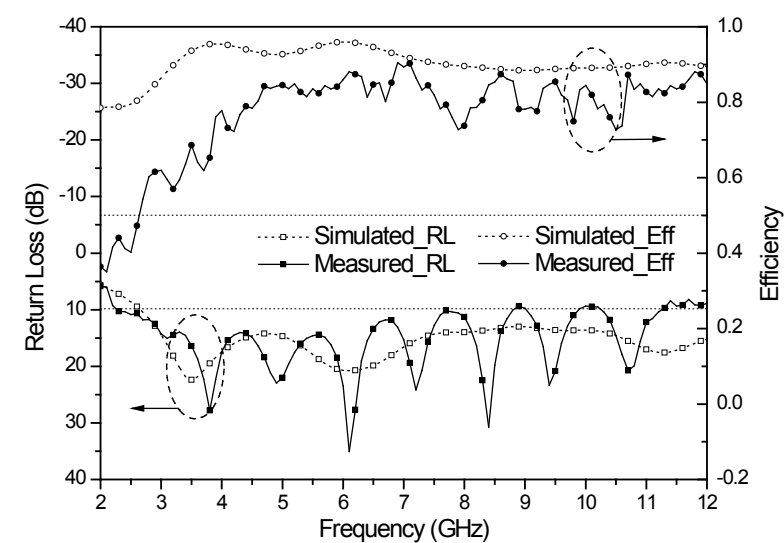

(g) $50(g l) * 30(g w)$

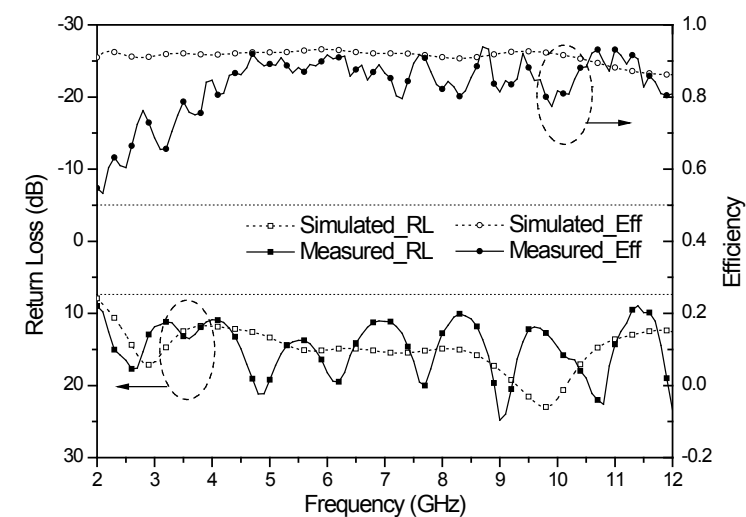

(h) $50(g l) * 50(g w)$

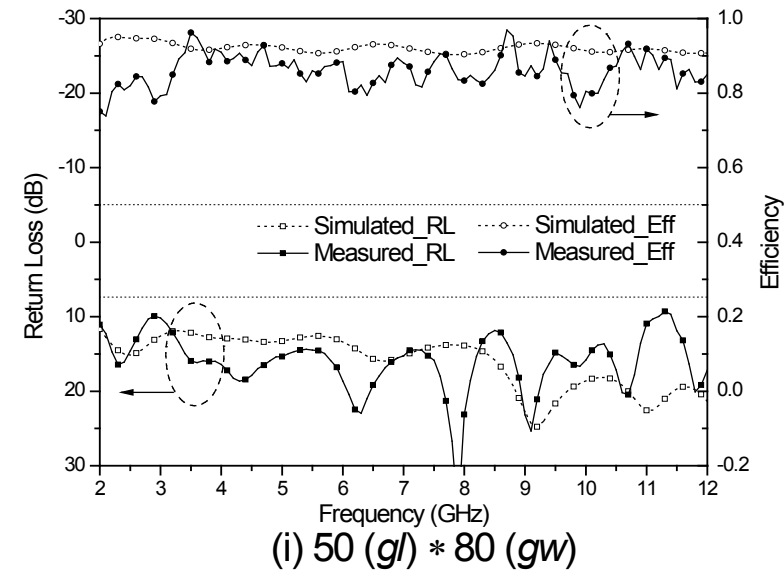

Fig. 3 Simulated and Measured Efficiencies and Return Loss of antennas with different ground plane sizes.

\section{CONCLUSIONS}

The effects of the dimensions of a rectangular ground plane on the performance of an elliptical monopole UWB antenna have been investigated using computer simulation and measurement. Results have shown that, the simulated and measured return losses agree well, but the measured efficiencies of the antennas are always less than the simulated efficiencies. The discrepancies are larger with smaller groundplane sizes and at lower frequencies. Moreover, the width of the ground plane has more effects on the efficiency than the length. Increasing the length of the ground plane lowers the lower cut off frequency of the antenna.

\section{REFERENCES}

[1] "Federal Communications Commission Revision of Part 15 of the Commission's Rules Regarding Ultra-Wideband Transmission Systems," FCC, 2002, First Report and Order FCC, 02.V48.

[2] J.T. Bernhard, J.J. Adams, M.D. Anderson, and J.M. Martin, "Measuring Electrically Small Antennas Details and Implications," iWAT 2009. International Workshop on Antenna Technology, 2-4 March 2009, pp. 1 -4 .

[3] T.W. Hertel, "Cable-current effects of miniature UWB antennas," Antennas and Propagation Society International Symposium, vol. 3A, pp. $524-527$, July 2005.

[4] K. G. Thomas and M. Sreenivasan, "A Simple Ultrawideband Planar Rectangular Printed Antenna With Band Dispensation," IEEE Trans. Antennas Propag., vol. 58, no. 1, pp. 27-34, Jan. 2010.

[5] H.D. Chen, "Ground plane effects on the microstrip-line-fed broadband sleeve monopole antennas", Microw.Antennas Propag.,Vol. 2, No. 6, pp. 601-605, 2008

[6] D.H. Kwon and Y. Kim, "Suppression of Cable Leakage Current for Edge-Fed Printed Dipole UWB Antennas Using Leakage-Blocking Slots," IEEE Trans. Antennas Propag., vol. 5, issue 1, pp. 183-186, Dec. 2006

[7] Z. N. Chen and T.S.P. See, "Small Printed Ultrawideband Antenna With Reduced Ground Plane effect," IEEE Trans. Antennas Propag., vol. 55, issue 2, pp. 383-388, Feb. 2007.

[8] Availanle on: http://www.cst.com. 